\title{
RADIATION FROM PLATINUM AT HIGH TEMPERATURES.
}

\author{
By G. K. Burgess
}

The study of the emissive properties of substances which can be brought to high temperatures without undergoing chemical or physical changes of surface is important in optical pyrometry for the practical realization and measurement of high temperatures; for a knowledge of the emissive properties of platinum, for instance, at various temperatures, gives a ready means of obtaining true temperatures from observations of the "black-body temperatures" as measured by an optical pyrometer. A platinum strip may replace to advantage an experimental black body, especially at temperatures above $1,500^{\circ} \mathrm{C}$, and may conveniently serve as a luminous source for the comparison or calibration of optical pyrometers.

It is the object of this paper to interpret the observations ${ }^{a}$ of Dr. Waidner and the author on the departure of platinum from blackbody radiation for red, green, and blue light, in terms of the now better known values of the higher temperatures involved, and as expressed by Wien's law as applied in a form first suggested by Lucas. ${ }^{b}$

For a black body, Wien's law for the distribution of energy in the spectrum may be written

$$
\log E=\log C_{1}-5 \log \lambda-\frac{C_{2} \log \varepsilon}{\lambda} \theta_{1}
$$

where $E$ is the energy radiated between wave lengths $\lambda$ and $\lambda+d \lambda, \theta^{\text {r }}$ the reciprocal of the absolute temperature, $C_{1}$ and $C_{2}$ constants, and $\varepsilon$, the logarithmic base.

For any other substance at the same photometric brightness we have

$a$ C. W. Waidner and G. K. Burgess: Optical pyrometry; Bull. Bureau of Standards, 1, p. 189; 1904.

$b$ R. Lucas: Phys. ZS., 6, pp. 19, 418; 1905. 


$$
\log E=\log C_{1}^{1}-n \log \lambda-\frac{C_{2}^{1} \log \varepsilon}{\lambda} \theta_{2}
$$

in which $n>5, C_{2}^{1}>C_{2}$ and $\theta_{2}>\theta_{1}$. Thus, for platinum $n=6.42$ according to Paschen, and $n=6$ from Lummer and Pringsheim's work. The largest value of $C_{2}$ for the visible spectrum is that deduced from the measurements of Lummer ${ }^{a}$ and Pringsheim: $C_{2}=5 \lambda_{m} T=5 \times 2940$ $=14700$, and the smallest value of $C_{2}^{1}$ is $C_{2}^{1}=6 \lambda^{1}{ }_{m} T^{1}=6 \cdot 2600=15600$.

That $\theta_{2}>\theta_{1}$, follows from the definition of a black body. $C_{1}$ and $C_{1}{ }^{1}$ are both small and the difference of their logarithms is negligible in most of what follows.

We may shorten the expressions by grouping the various constants, as follows:

$$
\text { (A) }\left\{\begin{array} { l } 
{ \operatorname { l o g } C _ { 1 } - 5 \operatorname { l o g } \lambda = K _ { 1 } } \\
{ \operatorname { l o g } C _ { 1 } ^ { 1 } - n \operatorname { l o g } \lambda = K _ { 1 } ^ { 1 } }
\end{array} \quad \text { (B) } \left\{\begin{array}{l}
C_{2} \frac{\log \varepsilon}{\lambda}=K_{2} \\
C_{2}^{1} \frac{\log \varepsilon}{\lambda}=K_{2}^{1}
\end{array}\right.\right.
$$

Whence, by equating (1) and (2),

$$
K_{1}-K_{2} \theta_{1}=K_{1}^{1}-K_{2}^{1} \theta_{2}
$$

Or, more simply,

$$
\theta_{1}=\alpha \theta_{2}+\beta
$$

Where

$$
\alpha=\frac{K_{2}^{1}}{K_{2}}=\frac{C_{2}^{1}}{C_{2}} \text { by }(B)
$$

And

$$
\beta=\frac{K_{1}-K_{1}^{1}}{K_{2}}=\frac{\lambda\left(\log C_{1}-\log C_{1}^{1}+(n-5) \log \lambda\right)}{C_{2} \log \varepsilon} \text { by }(A) \text { and }(B)
$$

or

$$
\beta=K \lambda \log \lambda
$$

where $K$ is a constant, characteristic of the substance.

Furthermore, the constants $\alpha$ and $\beta$ must lie within well-defined limits, as follows:

$$
\alpha>1 \text { since } C_{2}^{1}>C_{2}
$$

That is, $\alpha$ is always greater than unity, but approaches unity as the substance examined is more nearly a black body; $\alpha=1.06$ from

$a_{\mathrm{A}}$ discussion of the values of these constants is given by Lummer in Reports to Congress of Physics, Paris, 1900, vol. 2, p. 41. 
the data cited above. Again, $\alpha$ is independent of the wave length $\lambda$ to a first approximation, by (4).

Since experiment shows $n>5$, we have

$$
\beta>0
$$

or $\beta$ approaches zero as the black body condition is approached, and equation (5a) shows the dependence of $\beta$ upon the wave length, namely, that $\beta$ increases as the product of the wave length into its logarithm. The constant $n$ can evidently be computed by (5) if $\beta$ and $C_{2}$ are known, or conversely, $\beta$ may be calculated; but then, in these computations $\log C_{1}-\log C_{1}^{1}$ is no longer negligible.

Equation (3) was deduced by Lucas, ${ }^{a}$ although he does not show completely the interrelations of the constants involved nor the necessary limitations as to the numerical values of $\alpha$ and $\beta$.

Equation (3) with the above corollaries is fundamental and completely expresses the departure of any substance from a black body in terms of the temperature and two constants whose values are a measure of its emissive properties, and states that the reciprocals of the temperatures of a black body and any substance having the same photometric brightness are directly proportional.

As the equation is linear, a knowledge of the black body temperature and true temperature at two points only is sufficient to completely define the departure of the radiation of a given substance from that of a black body throughout the entire temperature scale, provided the substance undergoes no chemical change; and since $\alpha$ is a contant independent of $\lambda$, when equation (3) is determined for any single value of $\lambda$, that for any other value of $\lambda$ in the visible spectrum is had by an observation of $\theta_{2}$ at a single temperature. These facts are expressed by writing (3) in the form:

$$
\theta_{1}=\alpha \theta_{2}+K \lambda \log \lambda
$$

Experimetal verification of the above conclusions is had in the observations $^{b}$ of Dr. Waidner and the author on the relation between the black body temperature and the true temperature of platinum for wave lengths $\lambda=0.651 \mu$ (red), $\lambda=0.550 \mu$ (green), and $\lambda=0.474 \mu$ (blue). The observations were taken on a platinum strip mounted on a Joly meldometer, and the scale defined by melting points, that of platinum ${ }^{c}$ being assumed as $1,715^{\circ} \mathrm{C}$. and palladium (slightly impure) as $1,525^{\circ} \mathrm{C}$.

$a$ Lucas, 1. c.

$b$ Waidner and Burgess, 1. c.

$c$ Holborn and Henning: Sitzungber., Berlin Akad., March 2, 1905, p. 311. Harker: Chem. News, 91, pp. 262, 274; 1905. 
The accompanying table gives $S$, the black body temperatures (absolute) of the platinum as measured by a Holborn-Kurlbaum optical pyrometer, corresponding to the true absolute temperatures $T$. $\theta_{1}$ and $\theta_{2}$ are the reciprocal temperatures, $i$ e., $\theta_{1}=\frac{1}{S}$ and $\theta_{2}=\frac{1}{T}$, and the number of determinations of $S$ is given in the column headed $p$.

\begin{tabular}{|c|c|c|c|c|c|c|}
\hline \multicolumn{7}{|c|}{ Radiation from platinum. } \\
\hline \multicolumn{7}{|c|}{ RED LIGHT: $\lambda=0.651 \mu . \quad \theta_{1}=1.0256 \quad \theta_{2}+0.0000357$. } \\
\hline$T_{o b s}$ & $S_{o b s}$ & $\theta_{1}$ & $\theta_{2}$ & $T$ computed. & $T_{o b s \cdot}-T_{\text {calc }}$ & $p$ \\
\hline 996 & 939 & 0.0010650 & 0.0010040 & 996.3 & -0.3 & 6 \\
\hline 1055 & 990 & 10101 & 9479 & 1052.5 & +2.5 & 2 \\
\hline 1223 & 1145 & 8734 & 8177 & 1224.3 & -1.3 & 5 \\
\hline 1337 & 1246 & 8026 & 7480 & 1337.9 & -0.9 & 10 \\
\hline 1500 & 1392 & 7184 & 6667 & 1502.2 & -2.2 & 2 \\
\hline 1606 & 1482 & 6748 & 6227 & 1604.7 & +1.3 & 2 \\
\hline 1798 & 1647 & 6072 & 5562 & 1794.5 & +3.5 & 9 \\
\hline 1988 & 1814 & 5513 & 5030 & 1989.1 & -1.1 & 14 \\
\hline \multicolumn{7}{|c|}{ GREEN LIGHT: $\lambda=0.550 \mu . \quad \theta_{1}=1.0320 \theta_{2}+0.0000218$} \\
\hline 1337 & 1258 & 0.0007949 & 0.0007480 & 1334.9 & +2.1 & 5 \\
\hline 1500 & 1409 & 7097 & 6667 & 1500.3 & -0.3 & 2 \\
\hline 1606 & 1506 & 6640 & 6227 & 1607.0 & -1.0 & 2 \\
\hline 1798 & 1676 & 5967 & 5562 & 1795.2 & +2.8 & 4 \\
\hline 1988 & 1850 & 5405 & 5030 & 1989.6 & -1.6 & 10 \\
\hline \multicolumn{7}{|c|}{ BLUE LIGHT: $\lambda=0.474 \mu . \quad \theta_{1}=1.034 \theta_{2}+0.0500107$} \\
\hline 1606 & 1529 & 0.0006540 & 0.0006227 & 1607.3 & -1.3 & 2 \\
\hline 1798 & 1699 & 5886 & 5562 & 1789.3 & +8.7 & 2 \\
\hline 1988 & 1885 & 5305 & 5030 & 1989.2 & -1.2 & 8 \\
\hline
\end{tabular}

The observations for the red $(\lambda=0.651 \mu)$ satisfy the equation, see (3),

$$
\theta_{1}=1.0256 \theta_{2}+0.0000357
$$

the constants being determined by least squares. Similarly, for the green $(\lambda=0.550)$

$$
\theta_{1}=1.0320 \theta_{2}+0.0000218
$$


and for the blue $(\lambda=0.474)$

$$
\theta_{1}=1.034 \theta_{2}+0.0000107 \text {. }
$$

The values of $T$ as computed from the formula $T=\frac{\alpha}{\theta_{2}-\beta}$ are given in the fifth column of the table and $\Delta T$ in the sixth. The differences between the observed and computed values of $T$ are well within the errors of observation.

It will be seen, moreover, that within the same limits the value of $\alpha$ (coefficient of $\theta_{2}$ ) satisfies the necessary conditions of being slightly greater than unity (equation 6) and a constant nearly independent of the wave length (equation 4 ). The values of $\beta$ also satisfy the condition expressed in equations (5a) and (8).

Lucas $^{a}$ discusses Holborn and Kurlbaum's ${ }^{b}$ observations on platinum for red radiation $(\lambda=0.643)$, and finds they satisfy the relation

$$
\theta_{1}=0.9857 \theta_{2}+0.0000598
$$

with an average deviation of about $4^{\circ} \mathrm{C}$. Holborn and Henning ${ }^{c}$ find empirically for the same observations

$$
\theta_{1}=\theta_{2}+0.000507 \text {. }
$$

The preceding deductions from Wien's law are general and apply to any substance conserving the optical properties of its surface with continued beating. The comparative ease with which $\alpha$ and $\beta$ may be determined experimentally, and the simplicity of the linear relation connecting them, would seem to indicate the desirability of expressing mono-chromatic radiation properties in terms of these two constants $\alpha$ and $\beta$. To the same order of approximation, Planck's equation gives the same solution.

$a$ Lucas 1. c.

$b$ Holborn and Kurlbaum: Ann. d. Phys. 10, p. 225; 1903.

$c$ Holborn and Henning, l. c. 



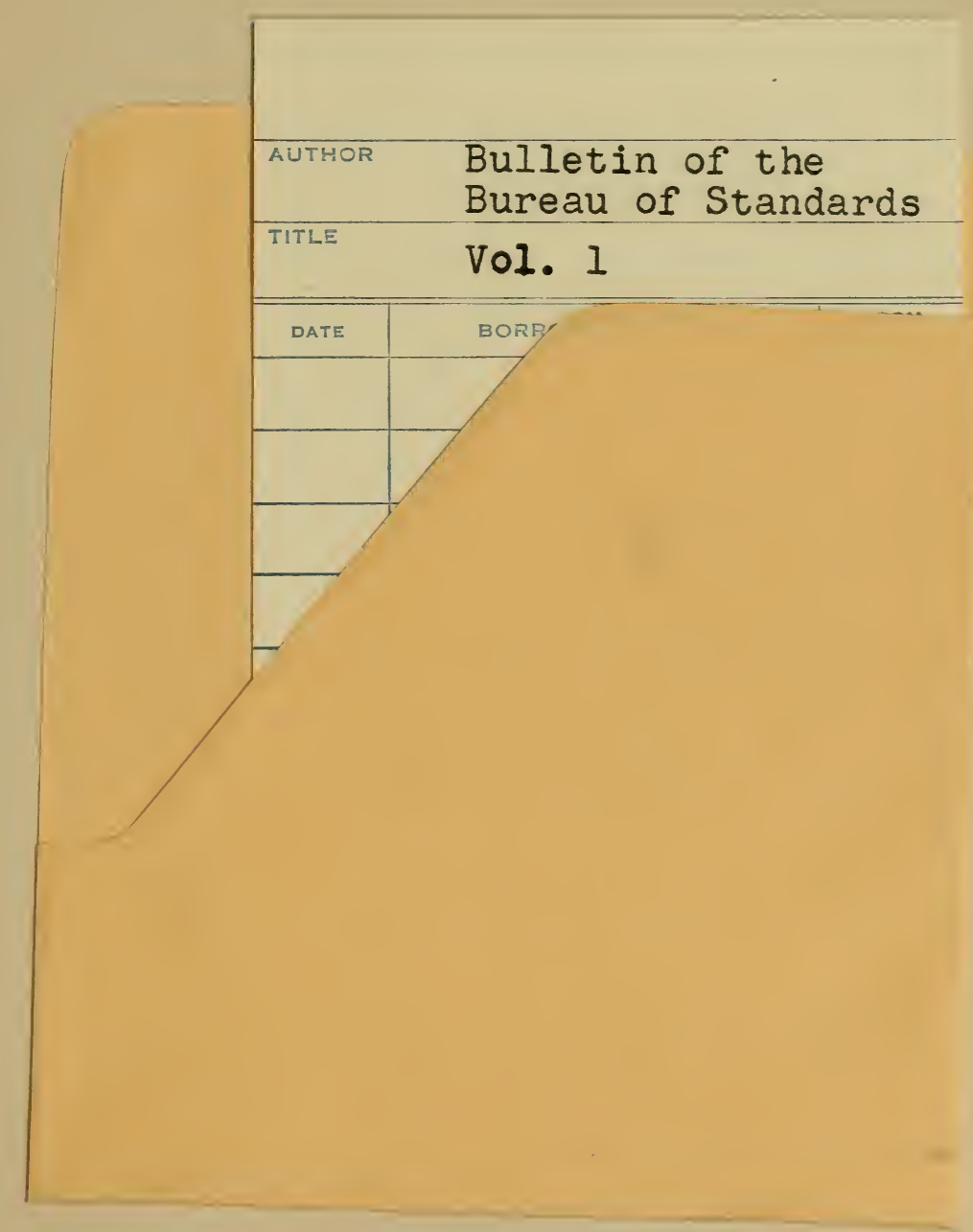


